\title{
Vestibular Asymmetry as the Cause of Idiopathic Scoliosis: A Possible Answer from Xenopus
}

\author{
François M. Lambert, ${ }^{1}$ David Malinvaud, ${ }^{1,2}$ Joan Glaunès, ${ }^{3}$ Catherine Bergot,${ }^{4}$ Hans Straka,,${ }^{1 *}$ and Pierre-Paul Vidal ${ }^{1 *}$ \\ ${ }^{1}$ Laboratoire de Neurobiologie des Réseaux Sensorimoteurs, Centre National de la Recherche Scientifique (CNRS) Unité Mixte de Recherche (UMR) \\ 7060 -Université Paris Descartes, 75006 Paris, France, ${ }^{2}$ Departement d'ORL et de Chirurgie Cervico-Faciale, Hôpital Européen Georges Pompidou, 75015 \\ Paris, France, ${ }^{3}$ Mathématiques Appliquées à Paris Descartes, CNRS UMR 8145-Université Paris Descartes, 75270 Paris cedex 06, France, and ${ }^{4}$ Laboratoire \\ de Radiologie Expérimentale, CNRS UMR 7052, 75010 Paris, France
}

\begin{abstract}
Human idiopathic scoliosis is characterized by severe deformations of the spine and skeleton. The occurrence of vestibular-related deficits in these patients is well established but it is unclear whether a vestibular pathology is the common cause for the scoliotic syndrome and the gaze/posture deficits or if the latter behavioral deficits are a consequence of the scoliotic deformations. A possible vestibular origin was tested in the frog Xenopus laevis by unilateral removal of the labyrinthine endorgans at larval stages. After metamorphosis into young adult frogs, X-ray images and three-dimensional reconstructed micro-computer tomographic scans of the skeleton showed deformations similar to those of scoliotic patients. The skeletal distortions consisted of a curvature of the spine in the frontal and sagittal plane, a transverse rotation along the body axis and substantial deformations of all vertebrae. In terrestrial vertebrates, the initial postural syndrome after unilateral labyrinthectomy recovers over time and requires body weight-supporting limb proprioceptive information. In an aquatic environment, however, this information is absent. Hence, the lesion-induced asymmetric activity in descending spinal pathways and the resulting asymmetric muscular tonus persists. As a consequence the mostly cartilaginous skeleton of the frog tadpoles progressively deforms. Lack of limb proprioceptive signals in an aquatic environment is thus the element, which links the Xenopus model with human scoliosis because a comparable situation occurs during gestation in utero. A permanently imbalanced activity in descending locomotor/posture control pathways might be the common origin for the observed structural and behavioral deficits in humans as in the different animal models of scoliosis.
\end{abstract}

\section{Introduction}

Adolescent idiopathic scoliosis (AIS) is one of the most familiar skeletal deformations in humans and manifested in its extreme form by a pronounced hunchback. However, the etiopathogeny is still unclear, largely due to its multifactorial origin (Weinstein et al., 2008). While a clear genetic basis is lacking so far (Miller, 2007), AIS is correlated in part with abnormal levels of tyrosine, sex and growth hormones, dysfunction of the melatonin-signaling pathway, and calcium-binding receptor protein calmodulin (Ahn et al., 2002; Moreau et al., 2004; Azeddine et al., 2007; Cheung et al., 2008). These biochemical abnormalities, however, might be more related to the progression rather than the origin of AIS (Kindsfater et al., 1994; Machida et al., 2009).

\footnotetext{
Received June 3, 2009; revised July 29, 2009; accepted Aug. 23, 2009.

Funding was provided by the French Centre National de la Recherche Scientifique and (entre National d'Etudes Spatiales. We acknowledge the help of Eric Pellé (Museum Nationale d'Histoire Naturelle de Paris) with the twodimensional X-ray imaging and the help of Barbara Vacca with data analysis. F.M.L. and D.M. performed the lesions and scanned the animals; C.B. provided support for the micro CT scans; J.G. reconstructed the skeletal images from the scans; H.S. and P.P.V. designed the study, developed the lesion protocol, and wrote this paper.

${ }^{*} H . S$. and P.-P.V. contributed equally to this work.

Correspondence should be addressed to Dr. Hans Straka, Laboratoire de Neurobiologie des Réseaux Sensorimoteurs, Centre National de la Recherche Scientifique Unité Mixte de Recherche 7060 -Université Paris Descartes, 45 rue des Saints-Pères, 75270 Paris cedex 06, France. E-mail: hans.straka@univ-paris5.fr.

DOI:10.1523/JNEUROSCI.2583-09.2009

Copyright $\odot 2009$ Society for Neuroscience $\quad$ 0270-6474/09/2912477-07\$15.00/0
}

A vestibular dysfunction has often been proposed as the trigger for AIS, but no causality has been shown so far (Herman et al., 1985; Rousie et al., 1999). An impaired postural and locomotor performance (Sahlstrand et al., 1979; Mallau et al., 2007), as well as deficits in extraocular motor control and gaze stabilization (Wiener-Vacher and Mazda, 1998; Rousie et al., 1999), including spontaneous and positional nystagmus (Jensen and Wilson, 1979; Sahlstrand and Petruson, 1979), was encountered in many patients. Most of these symptoms are compatible with a bilateral imbalance of the activity in central vestibular neurons (WienerVacher and Mazda, 1998; Manzoni and Miele, 2002). Nonetheless, it remains unclear whether a vestibular pathology is the common cause for the scoliotic syndrome as for the gaze/posture deficits or whether the latter behavioral deficits are a consequence of the scoliotic skeletal deformations.

Given the unclear origin of human AIS, various methods in different vertebrate species were used to induce scoliotic-like deformations (Barrios and Arrotegui, 1992; O'Kelly et al., 1999; Machida et al., 2006; Gorman and Breden, 2007). Pinealectomy triggered skeletal deformations in chicken (Machida et al., 1993; O'Kelly et al., 1999) and in rats in which the forelimbs had been surgically removed (Machida et al., 2005). Moreover, skeletal deformations were induced in rats by lesion of different motor control-related brainstem nuclei (Barrios and Arrotegui, 1992), suggesting asymmetric central nervous activity in descending pathways as a possible origin of scoliosis. An imbalance in activity 
is also provoked in vestibulo-motor circuits after an acute unilateral lesion of labyrinthine sensory inputs (Dieringer, 1995; Curthoys, 2000), which as a consequence causes severe perturbations of gaze and posture as well as abnormal limb positions (De Waele et al., 1989). However, in all terrestrial but not aquatic vertebrates, this syndrome recovers, largely due to sensory substitution of absent vestibular inputs by limb proprioceptive signals (Dieringer, 1995; Curthoys, 2000). Thus, our hypothesis was that an experimentally induced vestibular asymmetry in aquatic larvae of $\mathrm{Xe}$ nopus laevis would cause a persistent asymmetric tone in the axial and limb musculature. In the aquatic habitat it will be permanent because of the lack of limb proprioception, which as a consequence might cause permanent skeletal deformations, given that all bones develop under a continuous asymmetric mechanical tension. Preliminary results have been published in abstract form (Malinvaud et al., 2008).

\section{Materials and Methods}

All experiments were performed on the aquatic tadpoles and young adults of Xenopus laevis. After fertilization and an embryogenesis of $\sim 2 \mathrm{~d}$, animals hatch from the egg and develop through a series of continuously growing larvae within $\sim 2$ months to transform during metamorphosis into young adult frogs (Nieuwkoop and Faber, 1994). During premetamorphic larval stages, the successively appearing hind- and forelimbs grow continuously, while the tail is reduced during metamorphic climax. For the study, Xenopus tadpoles and young adults between stages 50-65 (Nieuwkoop and Faber, 1994) were subjected to a unilateral labyrinthectomy (UL; $n=15$ ) or to a combined removal of the horizontal and anterior semicircular canal ampulla $(n=4)$. The surgery was performed under general anesthesia with $0.03 \%$ MS-222 (3-aminobenzoic acid ethyl ester) in a frog Ringer solution [containing (in $\mathrm{mM}$ ) $75 \mathrm{NaCl}, 25 \mathrm{NaHCO}_{3}, 2$ $\mathrm{CaCl}_{2}, 2 \mathrm{KCl}, 0.5 \mathrm{MgCl}_{2}$, and 11 glucose, $\mathrm{pH}$ 7.4]. In all cases, the left otic capsule was opened and endorgans were removed under visual control.

Two-dimensional $X$-ray imaging. The skeleton of young adult frogs at stage 65-66 that received a UL either at larval stages or immediately after metamorphosis was visualized with an X-ray micro-scanner (Faxitron). After anesthesia in $0.03 \%$ MS-222 animals were put on a photographic film (Kodak industrex MX100) and exposed to brief low-voltage radiations (150 s, 20-25 KV). The X-ray films were developed by photographic processing and digitized with a CCD camera, equipped with a Nikon $60 \times$ objective. Scoliotic deformations of the spine in human patients are usually indicated by the Cobb angle (Griffet et al., 2000). For this purpose, landmarks on the spine of the frogs were selected and Cobb angles were automatically computed as the differences between maximum and minimal angles of the deviations from a vertical axis. A diffeomorphic matching between selected landmarks and landmarks positioned on a synthetic rectilinear vertebral column provided a general cost of deformation (Glaunès et al., 2004). In brief, the Measure-Based Diffeomorphic Matching (MBDM) allows to perform an optimal matching between two sets of points $X=\left(x_{1}, x_{2}, \ldots, x_{m}\right)$ and $Y=\left(y_{1}, y_{2}, \ldots, y_{n}\right)$, where the number of points $m$ and $n$ are possibly different and a correspondence between the points is unknown. In the present case, $X$ was the set of landmarks on the rectilinear column, and $Y$ the set of selected landmarks on the spine (see supplemental Fig. $1 A$, available at www. jneurosci.org as supplemental material). The applied method consists in finding an optimal deformation of the ambient space, $\varphi$ (visualized in Fig. $2 E, F$ via its action on a reference grid), which minimizes the function $J(\varphi)=E(\varphi)+D\left(\left(\varphi\left(x_{\mathrm{i}}\right)\right),\left(y_{\mathrm{j}}\right)\right)$, where $E(\varphi)$ describes the regularity of the deformation - constraining it to be a diffeomorphismand $D\left(\left(\varphi\left(x_{\mathrm{i}}\right)\right),\left(y_{j}\right)\right)$ describes the closeness between the source points transported by the deformation $\varphi\left(x_{\mathrm{i}}\right)$ and the target points $y_{\mathrm{j}}$ (see supplemental Fig. $1 A$, available at www.jneurosci.org as supplemental material). This measure of closeness involves the computation of all pairwise interactions between points through a Gaussian kernel $k(\mathrm{r})=\exp \left(-r^{2} / \sigma^{2}\right)$, where $\sigma$ is a fixed scale parameter: in other words, $D\left(\left(\varphi\left(x_{\mathrm{i}}\right)\right),\left(y_{\mathrm{j}}\right)\right)$ is a combination of all $k\left(\left\|\varphi\left(x_{i}\right)-y_{j}\right\|\right)$ for $i=1 . . m$ and $j=1 . . n$, where $\|$.$\| is the$ Euclidean norm (the usual distance between points). At the end of the matching process, the quantity $E(\varphi)$ gives an overall cost of deformation between the point sets.

Three-dimensional imaging and reconstruction. Some animals $(n=3)$ were used for a three-dimensional (3D) visualization of the skeleton in a micro CT-scan (SkyScan 1172 micro-CT X-ray, Microtomograph, SkyScan) for $3 \mathrm{D}$ imaging and subsequent reconstruction. Animals were deeply anesthetized by immersion in $0.03 \%$ MS-222 for $\sim 5 \mathrm{~min}$. To avoid positional changes during data acquisition, they were mechanically stabilized by putting them with the ventral half of the body in $3 \%$ agarose, leaving the dorsal side free. The agarose block with the frog in a vertical position was then arranged in a plastic tube with a volume of $\sim 12 \mathrm{~cm}^{3}$ filled with $0.03 \%$ MS-222 in water that maintained the deep anesthesia throughout the scanning process. Acquisition parameters for the scanning were: $40-50 \mathrm{kV}, 100 \mathrm{~mA}$, step rotation of $1^{\circ}$, large camera pixel resolution, duration $\sim 23 \mathrm{~min}$; reconstructions were made with the automatic data processing system from Skyscan 1172. 3D reconstructed volumetric images were processed by OsiriX software (OsiriX DICOM Viewer, version 3.5.1; The Osirix Foundation) to extract iso-surface meshes of the skeleton. Five landmarks were manually selected for each vertebra on the $3 \mathrm{D}$ mesh. The MBDM method described above was also applied to measure the $3 \mathrm{D}$ cost of deformation. For measurements of the asymmetry of each vertebra, slices of the $3 \mathrm{D}$ mesh were extracted by sectioning in a plane perpendicular to the vertebral column. On each reconstructed optical section, a symmetry transformation of the left half to the right half of a given vertebra along the midline was performed and the MBDM method was applied to match the superimposed sides of the vertebrae (supplemental Fig. $1 B$, available at www.jneurosci.org as supplemental material). Finally the asymmetry was quantified by determining the number of pixels of the right half of a vertebra, which had no corresponding pixel on the left half within a vicinity of $0.1 \mathrm{~mm}$. A division by the total number of pixels resulted in a value between 0 and 1 with 0 indicating symmetry and 1 asymmetry. All computations were done using Matlab software (MATLAB version 7.4.0.: The MathWorks, 2007).

\section{Results}

UL with a removal of all endorgans as well as Scarpa's ganglion was performed at different pre- and post-metamorphic stages in Xenopus laevis (see Material and Methods). The stage of the lesion was critical since it influenced the progression of the further ontogenetic development. In particular, a UL performed between stages 50-55 stopped further growth and largely arrested the animal at the developmental stage at which the lesion was performed. Only after the animals reached stage 56, a UL did not prevent tadpoles from further development although body growth was slower than in controls. Starting immediately after the surgery, all tadpoles exhibited a curvature of the body/tail toward the side of the lesion (Fig. $1 A$ ) that was caused by a relatively weaker contraction of the axial body musculature on the intact side (Fig. $1 F$ ). The bending of the body/tail as well as the accompanying circling movements did not cease throughout further development. After metamorphosis these animals consistently exhibited as young adult frogs (stage 65-66) a scoliotic postural syndrome (compare distorted with control animals in Fig. $1 B-E$ ) that was accompanied by strong deformations of the spine and all vertebrae (Fig. $2 C, D, F$ ). If the UL was performed early after metamorphosis, the typical postural syndrome (Fig. $1 D, E)$ known from other tetrapods (Dieringer, 1995; Curthoys, 2000) and the severe perturbations of the locomotor pattern was observed as well, however, clear skeletal deformations were absent as revealed by X-ray imaging (see below). These results suggest that there are two constraints for the induction of skeletal asymmetries in larval Xenopus. First, there is a developmental threshold for the induction of the skeletal deformations by a unilateral vestibular lesion. Before stage 56 symmetrical vestibular sensory signals are apparently indispensable for body growth. And second, the absence of skeletal deformations following a UL 

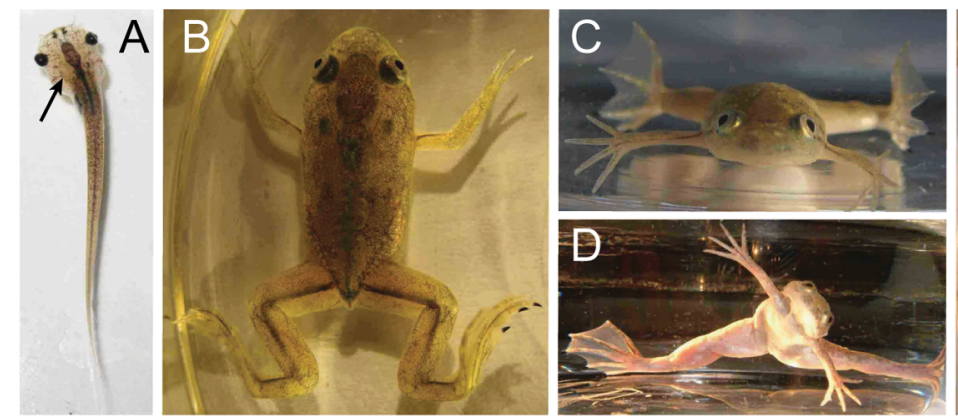

$\mathrm{F} 1$

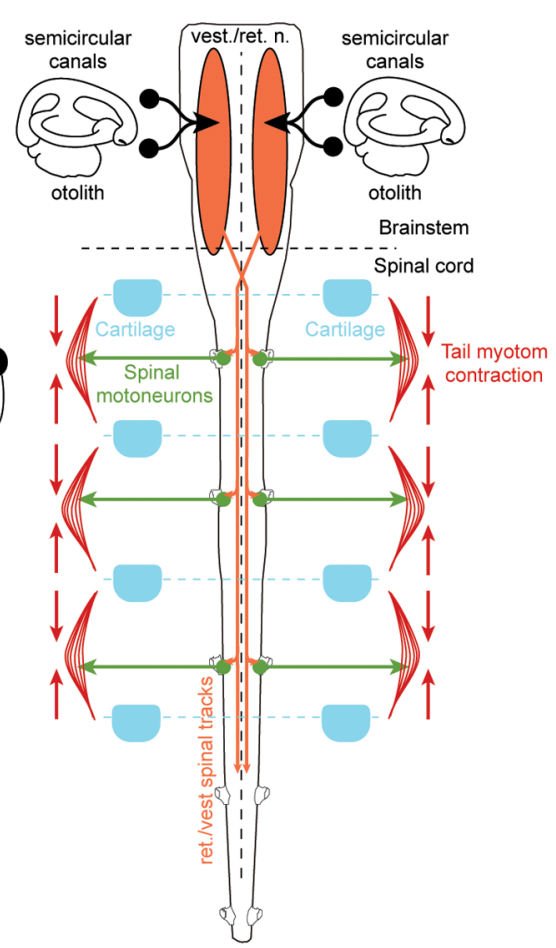

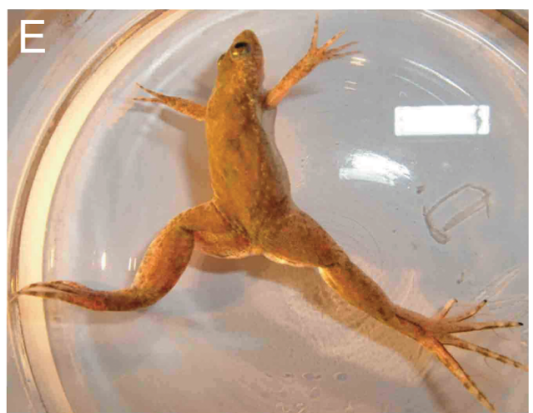

F2

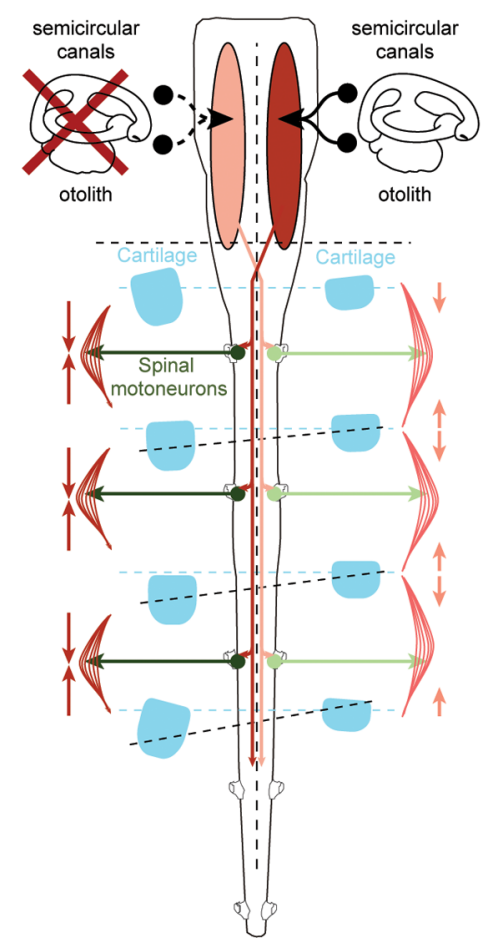

Figure 1. Postural deficits after a UL. $\boldsymbol{A}$, Top view of a stage 56 larval Xenopus laevis after removal of the left labyrinth (arrow), illustrating the induced bending of the body/tail. $\boldsymbol{B}-\boldsymbol{E}$, Top and front views of a stage 65 young adult control frog $(\boldsymbol{B}, \boldsymbol{C})$ and after $U \mathrm{~L}(\boldsymbol{D}, \boldsymbol{E}) . \boldsymbol{F}$, Schematic drawing depicting the organization of descending vestibulo-spinal/vestibulo-reticulo-spinal, spinal motor activity, contraction of axial musculature, and relative orientation of cartilaginous skeletal elements in controls $\left(\boldsymbol{F}_{\boldsymbol{1}}\right)$ and after a UL on the left side $\left(\boldsymbol{F}_{2}\right)$.

in young adult frogs suggests that after metamorphosis persisting asymmetric descending signals that cause an asymmetric muscular tone fail to twist the skeleton. This is likely because the individual skeletal elements are already highly ossified (Miura et al., 2008), and thus much more torsion-resistant due to a constant asymmetric muscular tone.

Changes in body symmetry and the induction of deformations in skeletal geometry, after a UL at larval stages 56-59 $(n=$ 5 ) were quantitatively analyzed by X-ray imaging of the skeleton after these animals developed into young adults. The skeletal deformations were quantified by measurements of the angle of the spine curvature, the Cobb angle (Fig. $2 B, D$ ), classically used in medical descriptions of the skeleton of scoliotic patients (Griffet et al., 2000). Moreover, the MBDM method was applied to compute an optimal deformation map (visualized via the deformed grids in Fig. 2E,F), and defined a mechanistic cost of the deformation. Even though the extent of the spine curvature varied slightly in the frontal plane between different animals it was consistently directed toward the ipsilesional side (Fig. 2C,D). This deformation was accompanied by a transverse rotation along the longitudinal body axis (Fig. 2C), as well as substantial deformations of the vertebrae. Spine curvature angles in young adult $\mathrm{Xe}$ - nopus that had received a UL at pre-metamorphic stage 56-59 $\left(44.6^{\circ} \pm 6.0^{\circ} ; n=5\right)$ were significantly larger ( $p \leq 0.001$, MannWhitney $U$ test $)$ compared with those of controls $\left(0.2^{\circ} \pm 3.6 ; n=\right.$ 8 ) or to those of frogs that received a UL after metamorphosis at stage $63-65\left(-0.6^{\circ} \pm 6.1 ; n=4\right)$ (Fig. $\left.2 G, H\right)$. The large spine curvature angles of the first group of animals were also accompanied by a significant change in the cost of deformation $(34.5 \pm 3.6)$ compared with controls (Fig. $2 G$ ) or with respect to the group of young adults $(9.77 \pm 0.81)$ that received a UL after metamorphosis (Fig. $2 G, H$ ). In general, the observed lesion-induced changes closely resembled the overall structural deformations observed in human idiopathic scoliosis. Based on micro-computer tomographic (CT) scan reconstructions of the entire skeleton (see Material and Methods; supplemental Movie 1, available at www. jneurosci.org as supplemental material), a 3D analysis of the structural asymmetries with a matching grid revealed a significant difference ( $p \leq 0.05$; Mann-Whitney $U$ test) in the deformation cost in all spatial orientations (Fig. 3) between stage 65 Xenopus $(180.0 \pm 44.1 ; n=3)$, which were exposed to a UL at pre-metamorphic stage 56-58, and age-matched control animals $(41.7 \pm 15.2 ; n=3)$. After landmark positioning on the same identifiable structures on each one of the 8 vertebrae, projected 
optical slices of the vertebrae were extracted (Fig. 3C,D) and submitted to a mathematical $3 \mathrm{D}$ rescaling. This procedure allowed obtaining the degree of rotation of all individual vertebrae with respect to the coronal plane (Fig. 3D, angle $\alpha$ ) as well as the rotation of the trigonometric axis of the vertebrae relative to the two axis of the coronal plane (Fig. 3D, angle $\beta$ ). The deviation from a mirrorimage symmetry of each vertebra allowed obtaining a quantification of the distortion of these skeletal elements (Fig. 3E, F; see supplemental Fig. $1 B$, available at www.jneurosci.org as supplemental material). These measurements revealed that the majority of the vertebrae are deformed in a lesion-typical directional manner that, however, was variable in its extent between different animals and dependent on the rostro-caudal position of the vertebrae along the spine (Fig. 3G). This analytical approach now allows comparing details of the vestibular lesion-induced deformations in frogs with those obtained from human scoliotic patients and moreover will also facilitate a better classification of the extent of scoliotic deformations in the latter.

Selective lesions of the horizontal and anterior canal ampulla without damage of other endorgans at pre-metamorphic larval stage $58(n=4)$ did not stop the further ontogenetic development, as did a complete UL, but induced a slight horizontal curvature of the body and tail toward the side of the lesion that disappeared after few days; as a consequence, post-metamorphic young adult frogs that were subjected to this particular lesion at their larval stage before metamorphosis did not exhibit a scoliotic syndrome and had Cobb angle values similar to those of controls (data not shown).

\section{Discussion}

Unilateral removal of the vestibular endorgans in larval Xenopus causes a distortion of skeletal elements after the animals continued to develop into young adults. The observed bilateral asymmetry in bone arrangement was similar to that seen in human AIS and was manifested in most cases as a pronounced hunchback. The causal relation between the induced vestibular asymmetry and the development of scoliotic skeletal deformations suggests that bilaterally balanced descending vestibular signals are essential for establishing a symmetrical body axis during ontogeny.

The present result in Xenopus clearly shows a causal link between a bilateral vestibular imbalance, the development of an asymmetric skeletal geometry and as a consequence a distorted body shape. The skeletal deformations of the spine and all vertebrae are highly reminiscent of idiopathic scoliosis in humans, including details such as Cobb angle values (Griffet et al., 2000). Although the skeletal deformations were induced by the entire removal of one labyrinth, it is likely that lesser deficits, in particular in otolith sensory inputs will also cause a persistent imbalance between bilateral central vestibular activity levels. Consequently, the establishment of an asymmetric skeletal geometry and body shape would not be an all-or-nothing phenomenon but graded and based on the extent of the imbalance. This concurs with the observation that the phenotype of body distortion and skeletal deformations in human AIS are also rather variable (Weinstein et al., 2008). Assuming a general validity of the results in Xenopus, the gaze/ posture deficits that accompany human AIS (Jensen and Wilson, 1979; Sahlstrand and Petruson, 1979; Sahlstrand et al., 1979; Byl et al., 1997; Wiener-Vacher and Mazda, 1998; Rousie et al., 1999; Manzoni and Miele, 2002; Mirovsky et al., 2006; Mallau et al., 2007) are compatible with a common vestibular origin, although a secondary influence of the distorted skeletal geome- 

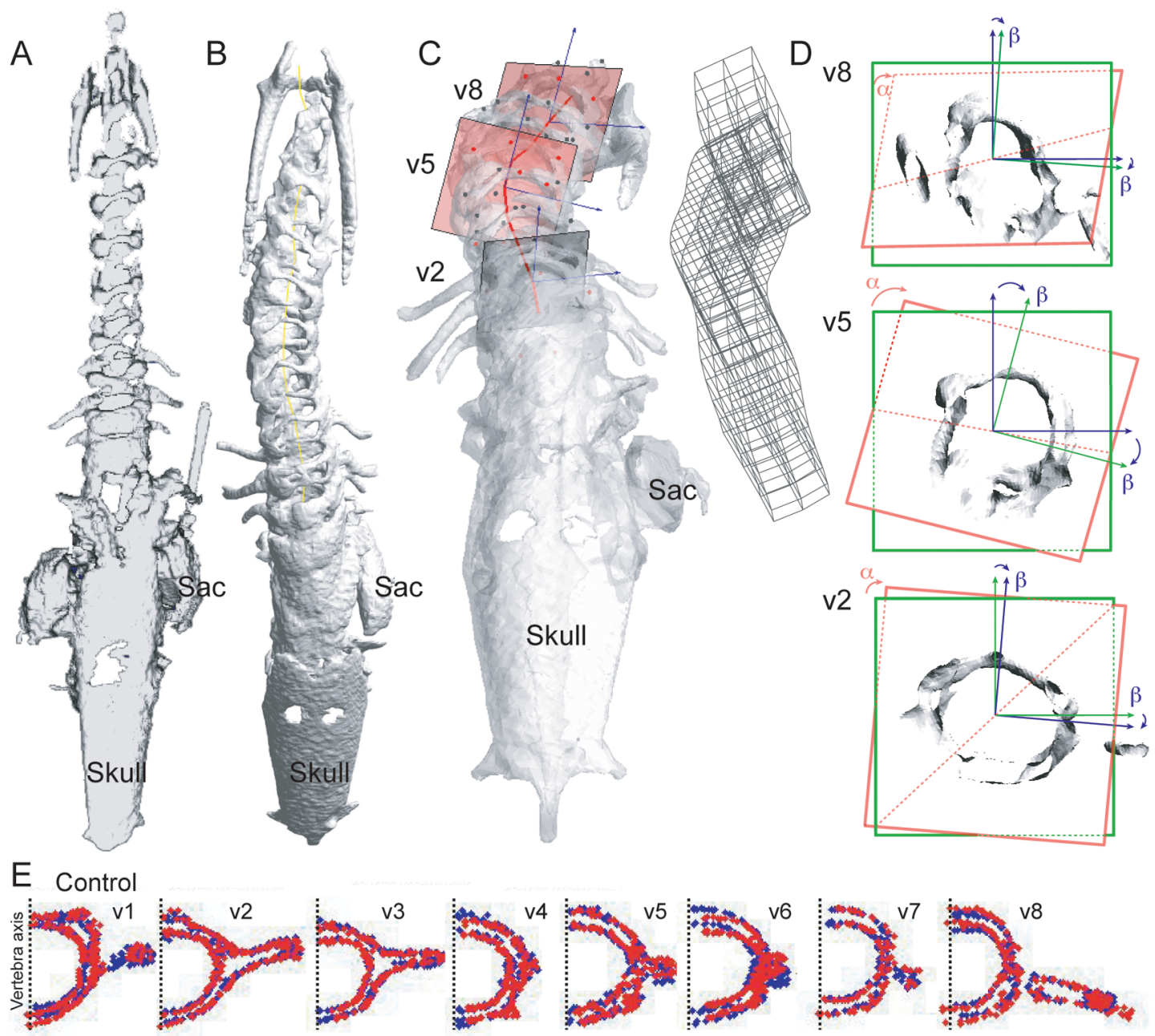

Pre-metamorphic labyrinth ablation

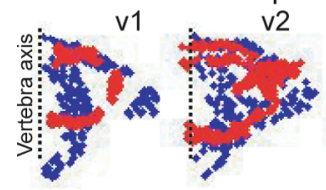

$\mathrm{F}$

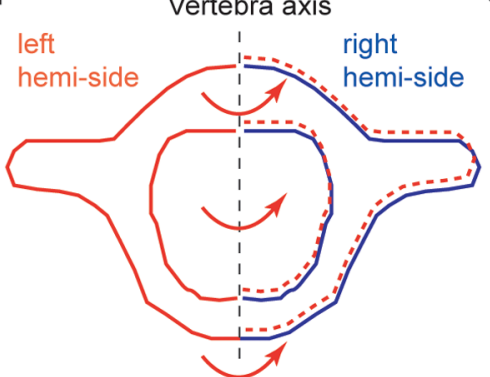

v3

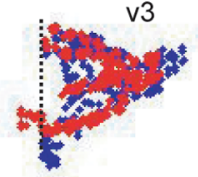

G

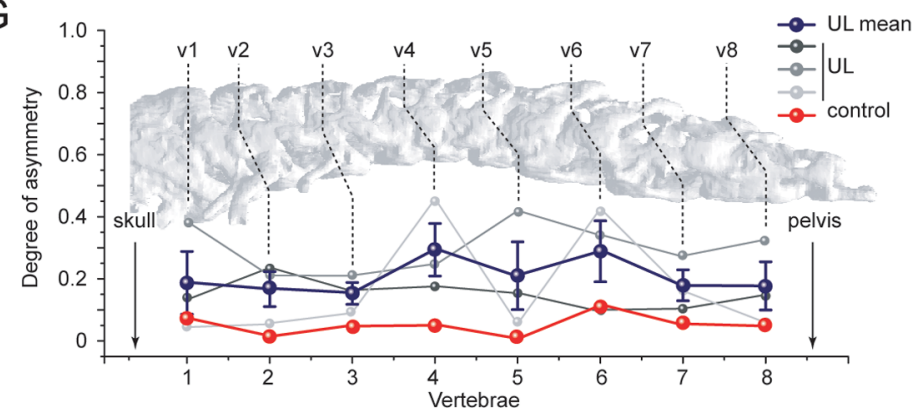

Figure 3. 3D quantification of the skeletal geometry in young adult Xenopus (stage 65-66). $A, B, 3 D$ reconstructed micro-CT scans of the vertebral column in a young adult control frog $(A)$ and a frog that received a UL at pre-metamorphic stage 56 (B).C, Optical extraction of 3 vertebrae (v2, v5, v8) and 3D grid of the deformation in a young adult UL frog. D, Plane and axis of rotation of v2, v5, and v8 in the coronal plane; $\alpha$ indicates the rotation of the actual frontal plane (red) of the vertebra relative to the theoretical coronal plane (green); $\beta$ indicates the rotation of the vertebral axis (green) relative to the dorsoventral axis (blue). $\boldsymbol{E}, \boldsymbol{F}$, Quantification of vertebrae (V1-8) symmetry in a young adult control frog ( $\boldsymbol{E}$, upper row) and in a frog that received a UL at pre-metamorphic stage 56 ( $\boldsymbol{E}$, lower row); blue dots indicate the outline of the right and red dots the left half of the vertebra which was optically flipped and superimposed on the right half $(\boldsymbol{F}) . \boldsymbol{G}$, Normalized degree of asymmetry $(0$, perfect symmetry between right and left side of the vertebra; 1, complete asymmetry) of individual vertebrae (v1-8) in a young adult control frog (in red) and three frogs that received a UL at pre-metamorphic stage 56 -58; the blue line indicates the mean of the $3 \mathrm{UL}$ frog; error bars are SE; dashed lines indicate the level of the vertebra along the rostro-caudal extent of the spine shown in the background.

try on extraocular/postural performance cannot be entirely ruled out. The development of skeletal asymmetries only after a lesion of the otolith organs in Xenopus complies with the notion that the utricle plays the major role in the control of the tone of the axial musculature, the establishment of symmetric skeletal components, and a symmetric body axis in humans (Wiener-Vacher and Mazda, 1998), as in guinea pigs (De Waele et al., 1989). 
The absent reports of skeletal distortions following experimentally induced vestibular lesions are likely related to the fact that mostly terrestrial vertebrates have been employed for these experiments (Ewald, 1892; Dieringer, 1995; Curthoys, 2000). These animals show a more or less complete recovery of the lesion-induced static postural deficits over time (Dieringer, 1995; Curthoys, 2000), facilitated by the multimodality of the vestibular system (Angelaki and Cullen, 2008). The recovery is a distributed process (Llinás and Walton, 1979) and involves cellular changes as well as sensory substitutions of the removed vestibular inputs in particular by forelimb proprioceptive signals (Straka et al., 2005). As a consequence, any asymmetric activity in vestibulo- and vestibulo-reticulo-spinal pathways after the lesion is not persistent. In contrast, in aquatic animals like larval and adult Xenopus, an asymmetric tonic neuronal discharge persists (Fig. $1 F_{2}$ ), since compensation of the postural deficits does not occur as indicated by the continuous rolling and circling movements of both tadpoles and young adults. This is most likely due to the lack of gravitation-related limb proprioception at larval stages (Fig. $1 A$ ) and after metamorphosis in young adults by their water-floating aquatic life style and the horizontal extension of the four limbs (Fig. $1 B, C$ ). Thus, the absence of body weightsupporting limb proprioception that could possibly substitute for absent vestibular sensory signals and thereby recalibrate the bilateral central activity in these animals is one of the necessary requirements to evoke the skeletal deformations by unilateral removal of the labyrinthine endorgans.

Moreover, vestibular lesions were usually performed in adult vertebrates with well developed highly ossified skeletons but not in embryonic or larval stages, as it was the case in the present study. In the Xenopus larvae used for the experiments, most skeletal elements were still cartilaginous and not, or only mildly ossified for a large part of the pre-metamorphic period (Miura et al., 2008). Under this condition, a continuous asymmetric activation of spinal motoneurons by descending activity will exert an asymmetric muscular pull (see Fig. $1 F_{2}$ ) that will likely distort the shape of the still flexible bones. The progressing ossification will then "fix" the skeletal elements in the deformed state during further development (Miura et al., 2008). Thus, at least two requirements were necessary to induce scoliotic deformations: the absence of body weight-supporting limb proprioceptive signals to recalibrate a vestibular-induced imbalance in descending motor pathways and rather soft skeletal elements that allow deformations by constant asymmetric muscular tone.

These particular conditions might link the present findings with human idiopathic scoliosis since similar conditions occur during human gestation. Developing human embryos as well as newborn babies during the first year have a rather limited use of limb proprioception that could be used as a reference frame to possibly recalibrate a vestibular imbalance (Assaiante et al., 2005). In addition, skeletal elements during this period would also be relatively soft and prone to deformations by a constant asymmetric pull of the inserting muscles. In the absence of systematic screening for deformed skeletal elements in newborns, scoliotic deformations are usually discovered only during early childhood (1-6 years), even though some of the extreme curvatures of the spine are detected at birth (Lincoln, 2007). Scoliosis, however, is only diagnosed if the spine curvature is $>10^{\circ}$ which is the case in $\sim 4 \%$ of the children (Dolan and Weinstein, 2007). Thus, unnoticed smaller deformations might cause an underestimation of this syndrome. Classically, the progression of idiopathic scoliosis subdivides into three periods: a slow phase until age 10-11 where forelimbs grow faster than the spine, a fast phase between age 12-16, considered as the highest risk period for severe deterioration, and a last phase until age 18, when the progression of the scoliotic deformations usually stops (Angevine and Deutsch, 2008). In general, an early diagnosis of a spine deformation $>19^{\circ}$ has an extremely high risk of further curvature progression, while smaller initial deformations improve and might become inconspicuous later, in part due to treatment (Weinstein et al., 2008). The huge predominance of diagnosed females with scoliosis $(\sim 5: 1)$ is likely related to a more pronounced progression through an impact of higher estrogen levels on factors that regulate bone growth during adolescence (Leboeuf et al., 2009).

The various biochemical and neurological pathologies that accompany human AIS as well as the different experimental animal models of scoliosis make it difficult to consolidate the existing results and to determine a clear origin of this syndrome. However, a permanent asymmetric activity in descending motor pathways to the spinal cord might be a common denominator. The results in Xenopus show that scoliotic deformations can be provoked by unilateral vestibular deficits that cause uncompensated, imbalanced activity in descending vestibulo-, reticulospinal, and other descending pathways affected by the removal of the vestibular periphery. This suggests that any functional asymmetry in descending pathways that causes substantial bilateral asymmetric activity of spinal motoneurons should produce similar structural deformations as the peripheral vestibular lesion. In fact, lesion of central pathways mediating proprioceptive signals in rabbits (Barrios et al., 1987) or lesion of brainstem nuclei related to postural control and equilibrium as the nucleus gracillis, the superior colliculus, or the lateral vestibular nucleus in rats was shown to provoke an imbalance of paraspinal muscle activity and kyphoscoliotic deformations (Barrios and Arrotegui, 1992). This result is compatible with the idea that destruction of these nuclei provokes an asymmetric activity in descending pathways similar to a peripheral vestibular lesion. The imbalanced activity after the central lesions is likely permanent since a recalibration would require morphological integrity of the destructed nuclei as shown for lesioninduced vestibular plasticity (Vibert et al., 1999).

Although the role of a melatonin-deficiency in the development of skeletal distortion is still unclear, pinealectomytriggered scoliosis is only evoked in bipedal rats in which the forelimbs had been surgically removed and the animals had learned to walk on their hindlimbs (Machida et al., 2005). An indirect impact of melatonin-deficiency on postural activity levels and muscle tonus through an effect on circadian rhythmgenerating brain structures as well as a direct melatonin-effect on posture control centers in the pontine tegmentum has been suggested to link pinealectomy with scoliotic deformations (Pompeiano et al., 2002). Assuming that pinealectomy also causes an imbalanced activity in descending motor control pathways, the asymmetry likely remains uncompensated in bipedal rats because of the absence of forelimb proprioception in contrast to normal rats (O’Kelly et al., 1999) or monkeys (Cheung et al., 2005), where pinealectomy fails to trigger skeletal deformations. A permanent asymmetric activity at different levels in posture/locomotionrelated descending pathways thus complies with vestibular-related brainstem disorders as a possible cause for human idiopathic scoliosis (Yamada et al., 1984; Herman et al., 1985) and suggests that unilateral labyrinthectomy in larval/adult Xenopus is an excellent model to further investigate issues related to this human disease. 


\section{References}

Ahn UM, Ahn NU, Nallamshetty L, Buchowski JM, Rose PS, Miller NH, Kostuik JP, Sponseller PD (2002) The etiology of adolescent idiopathic scoliosis. Am J Orthop 31:387-395.

Angelaki DE, Cullen KE (2008) Vestibular system: the many facets of a multimodal sense. Annu Rev Neurosci 31:125-150.

Angevine PD, Deutsch H (2008) Idiopathic scoliosis. Neurosurgery 63 [Suppl]:86-93.

Assaiante C, Mallau S, Viel S, Jover M, Schmitz C (2005) Development of postural control in healthy children: a functional approach. Neural Plast 12:109-118.

Azeddine B, Letellier K, Wang da S, Moldovan F, Moreau A (2007) Molecular determinants of melatonin signaling dysfunction in adolescent idiopathic scoliosis. Clin Orthop Relat Res 462:45-52.

Barrios C, Arrotegui JI (1992) Experimental kyphoscoliosis induced in rats by selective brain stem damage. Int Orthop 16:146-151.

Barrios C, Tuñón MT, De Salis JA, Beguiristain JL, Cañadell J (1987) Scoliosis induced by medullary damage: an experimental study in rabbits. Spine 12:433-439.

Byl NN, Holland S, Jurek A, Hu SS (1997) Postural imbalance and vibratory sensitivity in patients with idiopathic scoliosis: implications for treatment. J Orthop Sports Phys Ther 26:60-68.

Cheung KM, Wang T, Poon AM, Carl A, Tranmer B, Hu Y, Luk KD, Leong JC (2005) The effect of pinealectomy on scoliosis development in young nonhuman primates. Spine 30:2009-2013.

Cheung KM, Wang T, Qiu GX, Luk KD (2008) Recent advances in the aetiology of adolescent idiopathic scoliosis. Int Orthop 32:729-734.

Curthoys IS (2000) Vestibular compensation and substitution. Curr Opin Neurol 13:27-30.

De Waele C, Graf W, Josset P, Vidal PP (1989) A radiological analysis of the postural syndromes following hemilabyrinthectomy and selective canal and otolith lesions in the guinea pig. Exp Brain Res 77:166-182.

Dieringer N (1995) 'Vestibular compensation': Neural plasticity and its relations to functional recovery after labyrinthine lesions in frogs and other vertebrates. Prog Neurobiol 46:97-129.

Dolan LA, Weinstein SL (2007) Surgical rates after observation and bracing for adolescent idiopathic scoliosis: an evidence-based review. Spine 32 [Suppl]:S91-S100.

Ewald JR (1892) Physiologische Untersuchungen über das Endorgan des N. Oktavus. Wiesbaden: Bergmann.

Glaunès J, Younes L, Trouvé A (2004) Diffeomorphic matching of distributions: a new approach for unlabelled point-sets and sub-manifolds matching. Paper presented at the 2004 IEEE Computer Society Conference on Computer Vision and Pattern Recognition, Washington, DC, July.

Gorman KF, Breden F (2007) Teleosts as models for human vertebral stability and deformity. Comp Biochem Physiol C Toxicol Pharmacol 145:28-38.

Griffet J, Leroux MA, Badeaux J, Coillard C, Zabjek KF, Rivard CH (2000) Relationship between gibbosity and Cobb angle during treatment of idiopathic scoliosis with the SpineCor brace. Eur Spine J 9:516-522.

Herman R, Mixon J, Fisher A, Maulucci R, Stuyck J (1985) Idiopathic scoliosis and the central nervous system: a motor control problem. Spine 10:1-14.

Jensen GM, Wilson KB (1979) Horizontal postrotatory nystagmus response in female subjects with adolescent idiopathic scoliosis. Phys Ther 59: $1226-1233$

Kindsfater K, Lowe T, Lawellin D, Weinstein D, Akmakjian J (1994) Levels of platelet calmodulin for the prediction of progression and severity of adolescent idiopathic scoliosis. J Bone Joint Surg Am 76:1186-1192.

Leboeuf D, Letellier K, Alos N, Edery P, Moldovan F (2009) Do estrogens impact adolescent idiopathic scoliosis? Trends Endocrinol Metab 20: $147-152$.

Lincoln TL (2007) Infantile idiopathic scoliosis. Am J Orthop 36:586-590.

Llinás R, Walton K (1979) Vestibular compensation: a distributed property of the central nervous system. In: Integration in the nervous system (Asanuma H, Wilson VJ, eds) pp 145-166. Tokyo: Igaku Shoin.

Machida M, Dubousset J, Imamura Y, Iwaya T, Yamada T, Kimura J (1993) An experimental study in chickens for the pathogenesis of idiopathic scoliosis. Spine 18:1609-1615.

Machida M, Dubousset J, Yamada T, Kimura J, Saito M, Shiraishi T, Yamagishi M (2006) Experimental scoliosis in melatonin-deficient C57BL/6J mice without pinealectomy. J Pineal Res 41:1-7.

Machida M, Saito M, Dubousset J, Yamada T, Kimura J, Shibasaki K (2005) Pathological mechanism of idiopathic scoliosis: experimental scoliosis in pinealectomized rats. Eur Spine J 14:843-848.

Machida M, Dubousset J, Yamada T, Kimura J (2009) Serum melatonin levels in adolescent idiopathic scoliosis prediction and prevention for curve progression - a prospective study. J Pineal Res 46:344-348.

Malinvaud D, Lambert FM, Chanoine C, Straka H, Vidal PP (2008) Scoliosis - a causal relationship with vestibular asymmetry: the Xenopus model. Soc Neurosci Abstr 34:367.14.

Mallau S, Bollini G, Jouve JL, Assaiante C (2007) Locomotor skills and balance strategies in adolescents idiopathic scoliosis. Spine 32:E14-E22.

Manzoni D, Miele F (2002) Vestibular mechanisms involved in idiopathic scoliosis. Arch Ital Biol 140:67-80.

Miller NH (2007) Genetics of familial idiopathic scoliosis. Clin Orthop Relat Res 462:6-10.

Mirovsky Y, Blankstein A, Shlamkovitch N (2006) Postural control in patients with severe idiopathic scoliosis: a prospective study. J Pediatr Orthop B $15: 168-171$.

Miura S, Hanaoka K, Togashi S (2008) Skeletogenesis in Xenopus tropicalis: characteristic bone development in an anuran amphibian. Bone 43: 901-909.

Moreau A, Wang DS, Forget S, Azeddine B, Angeloni D, Fraschini F, Labelle H, Poitras B, Rivard CH, Grimard G (2004) Melatonin signaling dysfunction in adolescent idiopathic scoliosis. Spine 29:1772-1781.

Nieuwkoop PD, Faber J (1994) Normal table of Xenopus laevis (Daudin): a systematical and chronological survey of the development from the fertilized egg till the end of metamorphosis. New York: Garland.

O’Kelly C, Wang X, Raso J, Moreau M, Mahood J, Zhao J, Bagnall K (1999) The production of scoliosis after pinealectomy in young chickens, rats, and hamsters. Spine 24:35-43.

Pompeiano O, Manzoni D, Miele F (2002) Pineal gland hormone and idiopathic scoliosis: possible effect of melatonin on sleep-related postural mechanisms. Arch Ital Biol 140:129-158.

Rousie D, Hache JC, Pellerin P, Deroubaix JP, Van Tichelen P, Berthoz A (1999) Oculomotor, postural, and perceptual asymmetries associated with a common cause. Craniofacial asymmetries and asymmetries in vestibular organ anatomy. Ann N Y Acad Sci 871:439-446.

Sahlstrand T, Petruson B (1979) A study of labyrinthine function in patients with adolescent idiopathic scoliosis. I. An electro-nystagmographic study. Acta Orthop Scand 50:759-769.

Sahlstrand T, Petruson B, Ortengren R (1979) Vestibulospinal reflex activity in patients with adolescent idiopathic scoliosis. Postural effects during caloric labyrinthine stimulation recorded by stabilometry. Acta Orthop Scand 50:275-281.

Straka H, Vibert N, Vidal PP, Moore LE, Dutia MB (2005) Intrinsic properties of vertebrate vestibular neurons: function, development and plasticity. Prog Neurobiol 76:349-392.

Vibert N, Bantikyan A, Babalian A, Serafin M, Mühlethaler M, Vidal PP (1999) Post-lesional plasticity in the central nervous system of the guineapig: a "top-down" adaptation process? Neuroscience 94:1-5.

Weinstein SL, Dolan LA, Cheng JC, Danielsson A, Morcuende JA (2008) Adolescent idiopathic scoliosis. Lancet 371:1527-1537.

Wiener-Vacher SR, Mazda K (1998) Asymmetric otolith vestibulo-ocular responses in children with idiopathic scoliosis. J Pediatr 132:1028-1032.

Yamada K, Yamamoto H, Nakagawa Y, Tezuka A, Tamura T, Kawata S (1984) Etiology of idiopathic scoliosis. Clin Orthop Relat Res 184:50-57. 